\title{
Erratum: "A Two-Dimensional Hydrostatically Equilibrium Atmosphere of a Neutron Star with Given Differential Rotation" [Pis'ma Astron. Zh. 26, 917 (2000); Astronomy Letters 26, 788 (2000)]
}

V. S. Imshennik and K. V. Manukovskiǔ

In the paper by V.S. Imshennik and K.V. Manukovskiǔ [Astron. Lett. 26, 788 (2000)], a misprint was made on page 794: Fig. 2 was given instead of Fig. 4 for the second time. Here, we present the correct Fig. 4.

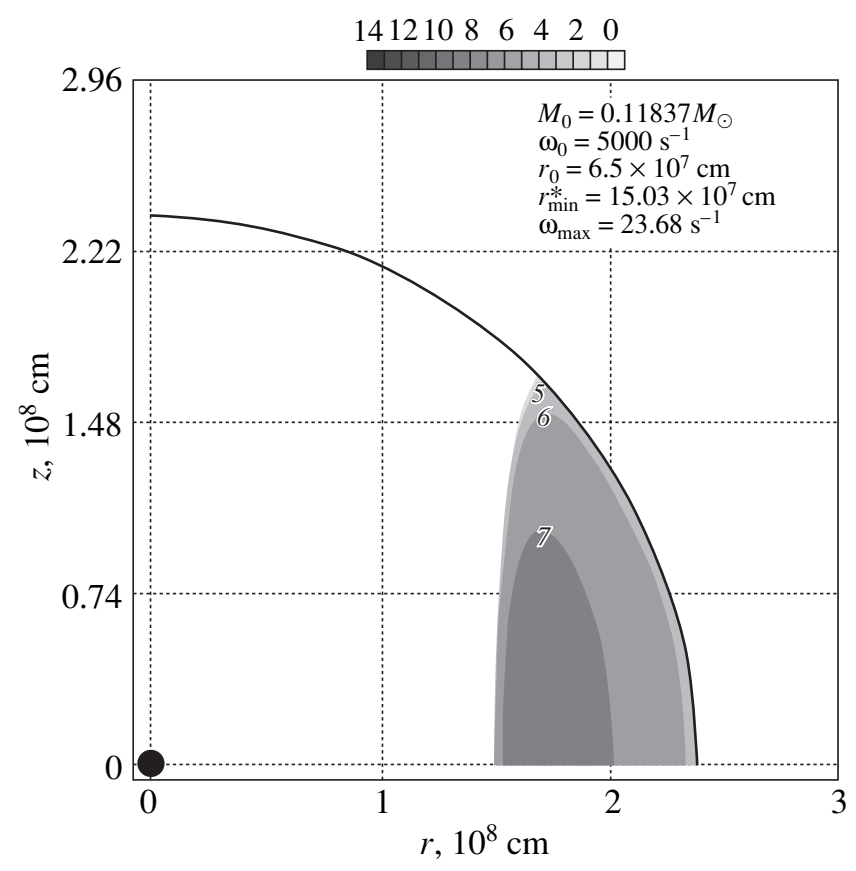

